METODOLOGÍA DE

LA INVESTIGACIÓN

Hernández Sampieri, Roberto;

Fernández-Collado, Carlos;

Baptista Lucio, Pilar

Mc Graw-Hill, México, 2006, 850 p.

\title{
DATOS DE LOS AUTORES
}

Roberto Hernández Sampieri es director del Centro de Investigación y Coordinación del Doctorado en Administración de la Universidad de Celaya.

Carlos Fernández-Collado es rector de la Universidad de Celaya. Profesor del Instituto Politécnico Nacional. Profesor Visitante de la Universidad de Oviedo.

Pilar Baptista Lucio es directora del Centro Anáhuac de Investigación, Servicios Educativos y Posgrado, de la Facultad de Educación en la Universidad Anáhuac.

\section{CONTENIDO}

El contenido de esta cuarta edición se divide en cuatro partes con un excelente desarrollo didáctico, apuntalado mediante la inclusión de esquemas que ilustran las explicaciones de cada sección.

La primera consta de dos capítulos. El primero de ellos, analiza las semejanzas y diferencias entre los dos enfoques investigativos en boga: el cuantitativo y el cualitativo; el segundo, desarrolla el procedimiento para la elaboración del proyecto de investigación en cualquier diseño, ya sea cuantitativo o cualitativo.

La segunda parte se dedica específicamente a la investigación cuantitativa, de manera que desarrolla los apartados que debe contemplar la labor heurística en este enfoque. Los capítulos de esta segunda parte 
llevan de la mano al lector desde el planteamiento del fenómeno, la búsqueda y la construcción del marco teórico, la formulación de hipótesis, hasta la elección del diseño idóneo de investigación, donde se privilegia el diseño experimental. Trata de manera menos prolija los diseños que los autores denominan no experimentales, donde incluyen algunos de carácter descriptivo y los estudios de caso. No faltan las indicaciones para determinar el tipo y tamaño de la muestra, cuestiones dudosas para la mayoría de los estudiantes que se inician en la investigación.

De esta misma segunda parte, es de recomendar el capítulo nueve, dedicado al diseño de los instrumentos de recolección de datos cuantitativos, donde se explicitan preceptos para la elaboración de cuestionarios y escalas para medir actitudes, tomando como base las escalas de medición y el análisis de contenido, de manera que no pueden faltar indicaciones para el análisis de la información recopilada. En el capítulo diez se desarrollan los elementos estadísticos - tanto descriptivos como inferenciales - y el apoyo de los programas electrónicos de análisis estadístico para realizar el tratamiento de los datos. Asimismo, ofrece indicaciones para lograr la confiabilidad y la validez. Este apartado finaliza con el reporte de los resultados de la investigación cuantitativa.

Por lo que toca a la tercera parte, constituida por seis capítulos, los autores desarrollan, de la misma manera detallada, el procedimiento para llevar a cabo una investigación cualitativa. Aquí se introduce al lector en la labor heurística cualitativa, donde conviene resaltar los criterios para la elección de la muestra en este tipo de enfoque, elemento que no es muy común encontrar en los textos de investigación. Se tratan, asimismo, los criterios para elaborar los instrumentos cualitativos de recolección de información, los diseños, el análisis de los datos cualitativos asistidos por computadora y finaliza con el reporte de investigación cualitativa.

La cuarta parte tiene como propósito mostrar la posibilidad de combinar ambos enfoques en la investigación del hecho educativo. 


\section{VALORACIÓN CRÍTICA}

La presente edición, la cuarta, modifica el contenido vertido en las ediciones anteriores, debido a que ahora desarrolla los tópicos relativos a la investigación cualitativa. Me parece conveniente señalar que el contenido del libro se sustenta en la primera parte —más precisamente en el primer capítulo, donde se tratan las «Similitudes y diferencias entre los enfoques cuantitativo y cualitativo»-, no obstante, a mi juicio, la taxonomía de la investigación presenta un problema conceptual, cuestión que es observable en el esquema de la página dos.

Dicho esquema divide los enfoques de la investigación de manera general en tres posibilidades, a saber: cuantitativo, mixto y cualitativo. Al referirse a las características del enfoque cuantitativo, incluye: «mide fenómenos, utiliza estadísticas, emplea experimentos, análisis causa-efecto». Al observarlas, es evidente que se refiere específicamente a un solo diseño, el experimental, puesto que las características enunciadas no corresponden a los estudios descriptivos. Considero que determinar las características en función de un diseño prototípico —el experimentalmanifiesta incongruencia con los diseños que no son experimentales.

Los diseños del enfoque cuantitativo incluidos en el texto - contemplados en el capítulo siete- como experimentales son: el experimento [propiamente dicho] y el cuasi experimento, y como no experimentales, las investigaciones: transeccional o transversal, longitudinal o evolutiva, longitudinales de tendencia y longitudinales de evolución de grupo, las cuales en realidad son diseños de investigación descriptiva. Además, incluyen en este mismo taxón a los estudios de caso y no se tratan los diseños ex post facto. Considero que la división de la investigación cuantitativa - experimentos y no experimentos- que los autores del libro asumen, puede causar confusión en los lectores poco experimentados.

A mi juicio, la investigación cuantitativa se divide por su propósito en causales y no causales, es decir, la primera responde a la pregunta ¿por qué suceden los fenómenos?, y la segunda a la pregunta ¿cómo suceden los fenómenos? Así las cosas, en los causales se incluyen los diseños: experimentales y no experimentales. A su vez, los experimentales están constituidos por los experimentos propiamente dichos, los pre experimentos y 
los cuasi experimentos. Por su parte, los no experimentales son los diseños ex post facto. En tanto que en los diseños no causales se ubican los descriptivos (esto es, los longitudinales, transversales, correlacionales, etcétera) debido a que la investigación descriptiva responde a la pregunta «¿cómo están sucediendo los fenómenos?» y no pretende llegar a explicaciones causales, donde una variable causa otra variable. Esta taxonomía permite la distinción de los diseños incluidos en el enfoque cuantitativo, debido a que los descriptivos en realidad son no causales, y considerarlos como no experimentales conduce al error de concebir que tengan el propósito de buscar relaciones de causa-efecto entre las variables y sólo se distinguen en que no son experimentales.

Por lo que toca a los estudios de caso, podemos encontrarlos en varios de los diseños: puede ser causal experimental en el experimento de caso único; también descriptivo ya sea longitudinal o transversal, por supuesto etnográfico o bien investigación evaluativa.

Por otro lado, para que las características abarquen a todos los diseños de la investigación cuantitativa, podemos considerar las siguientes: describe cómo o por qué suceden los fenómenos; mide cuantitativamente las variables; emplea la estadística (descriptiva e inferencial) para el análisis de los datos recopilados y tiene como propósito la generalización de los resultados obtenidos a la población de la que fue extraída la muestra.

A pesar de las cuestiones en las que difiero con respecto a la clasificación de la investigación de los autores, considero que el libro es un excelente compendio del proceder investigativo en el estudio del fenómeno educativo que pretenda seriedad, debido a que ofrece al lector el procedimiento de la labor heurística de cada diseño y le resuelve las dudas más comunes mediante ejemplos, además de ofrecerle una amplia gama de referencias bibliográficas que le permiten profundizar en el contenido deseado. 